\title{
Formes et fonctions du stéréotype dans des interactions en situations de contact
}

\section{Marion Perrefort}

\section{(2) OpenEdition \\ 1 Journals}

\section{Édition électronique}

URL : https://journals.openedition.org/aile/4917

DOI : 10.4000/aile.4917

ISSN : $1778-7432$

Éditeur

Association Encrages

\section{Édition imprimée}

Date de publication : 1 septembre 1996

Pagination : 139-154

ISSN : 1243-969X

\section{Référence électronique}

Marion Perrefort, «Formes et fonctions du stéréotype dans des interactions en situations de contact », Acquisition et interaction en langue étrangère [En ligne], 7| 1996, mis en ligne le 27 juin 2012, consulté le 14 avril 2022. URL : http://journals.openedition.org/aile/4917 ; DOI : https://doi.org/ 10.4000/aile.4917

Ce document a été généré automatiquement le 14 avril 2022.

(c) Tous droits réservés 


\title{
Formes et fonctions du stéréotype dans des interactions en situations de contact
}

\author{
Marion Perrefort
}

\section{NOTE DE L'ÉDITEUR}

Version remaniée d'une conférence faite avec B. Py au Centre de Recherche en Linguistique Etrangère de l'Université de Franche-Comté en février 1994.

1 En nous basant sur des séquences extraites de conversations bilingues et exolingues entre Allemands et Français, nous voulons décrire et analyser quelques procédés de stéréotypage et de gestion interactive des stéréotypes. Ces conversations ont été enregistrées lors de rencontres franco-allemandes, dans le cadre d'un projet de recherche interdisciplinaire sur la communication interculturelle sous l'égide de l'office Franco-Allemand pour la Jeunesse (OFAJ 1989 -1994) et auquel ont participé des étudiants germanistes français ( $\mathrm{LF}=$ locuteur français) et des romanistes allemands (LA = locuteur allemand) dont l'âge moyen était de 19 ans.

Deux remarques s'imposent quant à la particularité du corpus :

1. Il s'agit de rencontres entre groupes. L'effet de groupe contribue sans doute à une accentuation du stéréotypage et donne lieu à un processus de compétition entre les cultures dont les individus se font les représentants. Ils utilisent alors leur capital culturel et social pour se dépasser mutuellement dans la présentation la plus valorisante possible de leur culture et par conséquent d'eux-mêmes et pour accentuer les ressemblances à l'intérieur du même groupe et les différences avec l'autre (cf. Tajfel, 1982).

2. Nous avons choisi des exemples dans les enregistrements du début des rencontres. Non seulement les premières impressions ont tendance à amplifier les différences associées aux cultures en contact, mais c'est à l'ouverture d'une interaction que sont particulièrement concentrées et décisives "les négociations concernant les “images" et les "identités", les "places" et les "relations", ainsi que le protocole et l'enjeu global de l'échange: ces 
négociations "donnent le ton", et déterminent dans une certaine mesure la suite des opérations. » (Kerbrat-Orecchioni, $1984: 236)$.

3 Précisons que l'exploitation du vaste corpus réuni pendant les quatre ans qu'a duré le projet de recherche n'en est qu'à ses débuts et que l'analyse de l'évolution des stéréotypes et de leur modification éventuelle reste à faire.

\section{Définition et fonctions générales du stéréotype}

4 La notion de stéréotype sert à définir un système d'opinions, de convictions, de jugements et d'attitudes qui guide et structure la perception. Les caractéristiques du stéréotype sont, selon la plupart des auteurs (Lippmann, 1964; Bardin, 1980), la simplification exagérée, la généralisation et la fréquence. Le stéréotype se manifeste sous forme de clichés langagiers, de formules toutes faites, "qui gomme[nt] les particularités pour mettre en relief un aspect, hypertrophié dès lors. » (Fink, 1993).

5 Quasthoff cherche à décrire et à analyser le stéréotype avec les moyens de la linguistique et le définit comme « der verbale Ausdruck einer auf soziale Gruppen oder einzelne Personen als deren Mitglieder gerichteten Überzeugung.» (Quasthoff, 1973 : 28) Correspondant aux normes qui règlent les échanges quotidiens, il est une mesure d'économie, nécessaire dans les interactions sociales.

6 Le recours aux stéréotypes peut être considéré, sur le plan énonciatif, comme aide à l'activité langagière. En ce cas, les énoncés n'ont que très peu de contenu sémantique, comme le montrent les deux extraits suivants de lettres de soldats de la Guerre 14-18 (Perrefort, sous presse) $)^{1}$ :

\begin{tabular}{|l|l|}
\hline (1) & J'ai l'honneur aujourd'hui de vous faire savoir letat de mes nouvelles. \\
\hline (2) & $\begin{array}{l}\text { Je vient de recevoir votre carte qui ma fait un grand plaisir de recevoir de vos nouvelles don je } \\
\text { vois que vous aites en bonne sante aussi que votre mari }\end{array}$ \\
\hline
\end{tabular}

7 La reproduction des formulations stéréotypées échoue sur le plan syntaxique et ces échecs sont autant de traces de l'insécurité des scripteurs, lesquels, dans leur souci de se rapprocher du modèle, alignent maladroitement formule après formule. Mais pour ces scripteurs malhabiles, l'accès à l'activité langagière écrite se trouve néanmoins facilité grâce aux formules toutes faites.

8 Ces «kits langagiers » remplacent la propre créativité langagière tant au niveau expressif qu'au niveau cognitif, mais réduisent l'activité communicative à l'établissement et au maintien du contact social.

9 L'emploi de tels stéréotypes fortement normés est quasi automatique. De sorte qu'ils ont un haut degré de prédictabilité et obéissent à des règles fixes d'agencement, de combinaison et d'adéquation pragmatique.

10 Ainsi compris, le stéréotype fait partie intégrante de notre savoir socio-culturel concernant les règles implicites qui structurent nos échanges avec autrui.

11 En raison de leur force simplificatrice et réductrice, les stéréotypes jouent un rôle sémantique important dans les échanges quotidiens. Ainsi on ne peut comprendre 
l'énoncé « Ma belle-mère vient demain, mais elle est assez sympa » que si on connaît les stéréotypes courants associés dans la culture française à belle-mère.

Par ailleurs, cet énoncé suppose que l'allocutaire partage ce savoir avec le locuteur et attribue aux catégories évoquées les mêmes caractéristiques, c'est-à-dire qu'il procède au même stéréotypage.

Donc, la connaissance des stéréotypes est indispensable pour la reconstruction des processus d'intercompréhension et pour saisir la cohésion de nombreux énoncés.

Dans le contact interculturel, notre savoir quotidien perd son efficacité explicative. L'ordre sémiotique habituel est perturbé, s'effondre même. Les signes ne peuvent plus être décodés comme à l'accoutumée, il est difficile d'établir des relations référentielles, les inférences échouent, bref le système culturel perd sa pertinence en tant que modèle sémiotique et le sentiment d'«avoir perdu pied», la perte d'orientation, créent une situation d'anomie.

15 Comme cette situation est difficile à vivre, la nécessité s'impose de créer de nouveaux automatismes et de reconstruire des significations. Dans ces opérations d'interprétation, les catégorisations et les stéréotypisations qui y sont liées sont d'une importance toute particulière: d'une part, ils fonctionnent comme marqueurs d'identité et d'altérité et contribuent ainsi à stabiliser la conscience perturbée de soimême. L'insécurité linguistique et culturelle est sublimée par la reprise à son compte des stéréotypes circulant dans la culture d'origine au sujet de la culture étrangère: «Employés et reproduits au sein de la famille, dans les groupes de pairs, dans la vie associative, diffusés par les discours des médias, les stéréotypes véhiculent des croyances partagées par la plupart des membres de la communauté » (Lüdi, 1995 : 210).

16 D'autre part, ils aident à classer les impressions, à mettre de l'ordre dans les données de l'altérité et à leur donner un sens. Ils sont indispensables dans la quête de modèles de comportements adéquats.

17 Les représentations, les présupposés, les attributions stéréotypées vont affecter - du moins dans un premier temps - la perception et l'interprétation de l'altérité et " agissent comme des outils qui permettent de manipuler symboliquement la région d'accueil » (Oesch-Serra, 1995, cf. aussi Franceschini, Oesch-Serra et Py, 1990).

18 Nous terminerons ces réflexions d'ordre général, en proposant, aux fins de notre analyse, la définition suivante du stéréotype :

19 L'activité langagière contribue de manière décisive à la construction de la réalité et à la constitution identitaire. (Berger et Luckmann, 1992). Dans ce sens, le stéréotype est une construction discursive, un objet de discours qui remplit certaines fonctions dans les interactions. Il est actualisé et géré interactivement et contribue à définir le contexte.

20 Par ailleurs, il sert, en tant que catégorie sémantique, à l'orientation, à la classification et à la construction du sens. Dans des rencontres interculturelles, il remplit une fonction sémiotique particulièrement importante dans les opérations interprétatives et les catégorisations identitaires. A ce titre, le stéréotype fait figure de symbole à valeur identitaire, utilisé " with reference to concepts in the mind of the user; these symbols are the means by which we define ourselves and others » (Le Page et Tabouret-Keller 1985 : 248 ; cf. aussi : Lüdi, 1995).

21 Par stéréotypage, j'entends des techniques interactives qui visent à associer aux catégories évoquées, p. e les Allemands/Français, hommes/femmes, des activités, des 
caractéristiques ou jugements de valeur «typiques » $\mathrm{X}$ font/ont/sont $\mathrm{Y}$ et mettent ainsi en scène les catégories.

\section{Le stéréotypage de l'identité culturelle}

Chacun de nous dispose, dans les échanges quotidiens, non pas d'une seule catégorie identitaire, mais d'un réseau d'appartenances catégorielles multiples (Sacks, 1972). Selon le contexte, les enjeux, les relations que nous voulons établir, nous mettons en scène ces appartenances diverses pour décrire et organiser l'expérience subjective de notre environnement social et culturel. Le choix des attributions associées aux catégorisations identitaires reflétera, matérialisé dans les stéréotypes, des scénarios socio-culturels collectifs.

Dans une situation de contact interculturel, une catégorie fréquemment sollicitée est celle de l'appartenance culturelle - catégorie identitaire qui n'est pas particulièrement pertinente dans les échanges quotidiens et dont la gestion interactive peut s'avérer difficile.

4 La catégorisation de l'appartenance culturelle et du réseau des propriétés et des activités qui y sont liées se fait souvent de manière contrastive. La référence à une catégorie implique alors l'évocation explicite ou implicite de celle qui lui est opposée : la catégorie d'appartenance culturelle Français, par exemple, appellera son pendant, c'est-à-dire la catégorie d'appartenance culturelle Allemand :

\begin{tabular}{|l|l|l|l|}
\hline (1) & LF : & $\begin{array}{l}\text { non moi/en tant qu'Allemands je pense que vous êtes mieux informés que nous hein sur } \\
\text { tout ce qui a pu se passer (OFAJ, 1989) }\end{array}$ \\
\hline
\end{tabular}

Dans cette séquence, le procédé de catégorisation («Allemands») sert à classer les interlocuteurs en même temps comme "natifs ", " experts ", " informateurs » et, par effet de contraste, tend à accentuer l'asymétrie relationnelle: "natif vs non natif ", « expert vs non expert », etc.

Cette configuration peut constituer une menace pour les faces des partenaires (Goffman, 1973, Kerbrat-Orecchioni, 1992), par exemple pour la face positive du natif, au cas où il n'accomplirait pas la tâche qui lui incombe. Nous reviendrons plus loin sur les activités de figuration rendues nécessaires devant les menaces potentielles des faces mises en présence.

27 Un procédé inverse de celui que nous venons de voir consiste à mettre en avant des attributs ou des activités considérés comme typiques et à remonter, à partir de là, à la catégorie elle-même :

(2) LF : $\begin{aligned} & \text { aber am Mittagessen habe ich bemerkt, daß Sie keine Zeit haben und Sie sprechen nicht } \\ & \text { viel und eh es ist sehr schnell (OFAJ 90) }\end{aligned}$

28 En (2), l'attribution stéréotypée d'un trait culturel permettant de remonter à la catégorie «Allemand » est initiée par un élément négatif, qui appelle son complément positif. Celui-ci peut être rendu explicite à l'aide de la procédure d'expansion proposée 
par Labov et Fanshel (1977), ce qui donnerait à peu près ceci : «Contrairement à vous, les Allemands, nous, les Français, prenons le temps, nous discutons à table.... »

Chaque catégorisation s'accompagne d'attributions généralisantes et la perception d'une personne comme appartenant à une catégorie fait qu'on lui attribue souvent de façon exagérément stéréotypée toutes les caractéristiques associées à la catégorie en question en soulignant les caractéristiques divergentes avec ses propres appartenances. Le choix des attributions reflétera notamment des scénarios culturels collectifs. L'exemple (3) est extrait d'une conversation qui a eu lieu le premier jour d'une des rencontres. Dans cette séquence, les partenaires discutent des différences entre Français et Allemands, en particulier dans leur apparence physique. La participante allemande, dont LF1 et LF2 avaient entendu mais non retenu le nom lors de la séance de présentation la veille, s'appelait Britta :

\begin{tabular}{|l|l|l|}
\hline (3) & LF1 & $\begin{array}{l}\text { les Français ne mettent quasiment pas de sandales comme les Allemands mettent } \\
\text { comme euh Gerta }\end{array}$ \\
\hline & LF2: & Gre/euh \\
\hline LF1: & Greta \\
\hline LF2: & $\begin{array}{l}\text { c'est des trucs là moi je trouve ça berk } \\
\text { (OFAJ 1991) }\end{array}$ \\
\hline
\end{tabular}

La perception de certains attributs physiques et vestimentaires avait conduit à une catégorisation de la personne comme représentante prototypique de "l'Allemande ».

On remarque dans l'exemple précédent le processus de dénomination engagé par LF1 et LF2 qui ont recours à des stéréotypes sémantiques pour retrouver le prénom dont elles n'ont retenu que les phonèmes $/ \mathrm{r} / / \mathrm{a} / / \mathrm{t} /$.

En effet, elles puisent dans leur répertoire onomastique, lequel relève à son tour d'un savoir stéréotypé concernant les noms allemands dans le contexte culturel français, en l'occurrence l'image de la « Gretchen » allemande, si fréquente dans les caricatures ou autres documents iconographiques.

La perception acoustique du nom associée à la perception visuelle de certains signes statiques - morphotype, vêture, parure - (Kerbrat-Orecchioni, 1986) avait déclenché un processus de sémantisation dont la matérialisation discursive Greta permet d'accéder à une des représentations stéréotypées de la femme allemande chez les locuteurs français.

35 La séquence (4) met en évidence la fonction sémiotique du stéréotype. Lors de la première soirée d'une rencontre en France, la consommation d'alcool était particulièrement élevée. Le lendemain ce fait fut thématisé par les participants :

\begin{tabular}{|l|l|l|}
\hline (4) & LF1 & $\begin{array}{l}\text { ils boivent beaucoup les Allemands moi qui bois pas beaucoup de vin je bois/je trouve } \\
\text { que les filles comme les garçons }\end{array}$ \\
\hline & LF2 & ils boivent \\
\hline
\end{tabular}


Derrière la catégorisation contrastive - buveur allemand vs buveur français - se profile le stéréotype ancien du buveur allemand immodéré que décrit déjà Montaigne, et de son antonyme, le buveur français modéré, possédant selon R. Barthes la maîtrise du boire puisque «savoir boire est une technique nationale qui sert à qualifier le Français à prouver à la fois son pouvoir de performance, son contrôle et sa sociabilité » (Barthes, 1957).

L'exemple (4) permet non seulement d'illustrer la fonction du stéréotype comme moyen économique de la pensée et comme grille explicative, mais les constructions collectives du stéréotype dans les séquences (3) et (4) mettent aussi en évidence sa fonction stabilisatrice pour la cohésion du groupe culturel, voire sa force coercitive.

Nous trouvons en effet de nombreuses séquences où l'ensemble des locuteurs réalisent le stéréotypage, soit à l'aide d'une énonciation collective, en choeur (stéréotypage homophonique), pour ratifier, renforcer ou, au contraire, refuser des affirmations avancées par les partenaires, soit en polyphonie (stéréotypage polyphonique) par des reprises partielles d'une contribution, des extensions, des achèvements, effectués tour à tour par deux ou plusieurs interlocuteurs.

En (6), les locuteurs français avaient demandé aux locuteurs allemands ce qu'ils pensaient des formes de salutations en France, en particulier de la «bise » :

\begin{tabular}{|l|l|l|}
\hline (6) & LA2 & weiss ich gar nicht die Begrüssung is ja auf jeden Fall schon mal persönlicher \\
\hline & LA1 & ja \\
\hline & LF1 & bitte ? \\
\hline & LA2 & die Begrüssung hier in Frankreich ist persönlicher \\
\hline & LF1 & als in Deutschland \\
\hline & LA2 & ja \\
\hline & LF1 & wieso ? \\
\hline & LA2 & denn es is doch was anderes ob du jemanden auf die Wange küsst oder du ihm die Hand \\
einfach nur gibst
\end{tabular}


40 À travers l'auto-stéréotypage négatif (« la froideur allemande »), les locuteurs allemands se distancient collectivement d'une pratique de leur propre culture pour juger d'autant plus positivement les habitudes culturelles françaises.

41 Le stéréotypage polyphonique renforce la position haute des locuteurs natifs - surtout s'il s'agit d'un auto-stéréotypage valorisant - et fragilise la position de l'alloglotte, qui n'a souvent pas les moyens linguistiques pour réagir de manière adéquate. C'est le cas dans l'exemple (7).

42 Selon les locutrices françaises les femmes allemandes n'attachent pas beaucoup d'importance à leur apparence et, en particulier, elles ne s'épilent pas les jambes :

\begin{tabular}{|l|l|l|}
\hline (7) & LF1 & qu'est ce que t'en penses toi \\
\hline & LA & de la vom rasieren oder was \\
\hline & LF1 & oui les femmes qui se rasent pas \\
\hline & LA & c'est mieux (rit) \\
\hline & LF & (toutes) grands cris de protestation/ \\
\hline & LF2 & je vais lui montrer mes jambes \\
\hline & LA & wenn se sich rasieren \\
\hline & LF & (toutes) ah ah ah ah \\
\hline & LA & je je je pense äh aussi que c'est un peu dégoûtant que \\
\hline & LF & rires \\
\hline & LF & que les femmes ähm ähm \\
\hline & LF1 & se négligent \\
\hline & LA & oui \\
\hline & c'est vrai (OFAJ 89) \\
\hline
\end{tabular}

Le stéréotypage collectif conjugué avec l'insécurité linguistique de l'alloglotte (ici LA) finit par engendrer chez ce dernier une attitude distanciée vis-à-vis du trait stéréotypé stigmatisé. Notons le large consensus implicite entre les membres du même groupe. Puisant dans le savoir commun, elles semblent connaître à l'avance et sans concertation aucune les réponses qu'il convient de donner. 


\section{La gestion des stéréotypes}

\section{interprétations
(Perrefort 1990).}

d'une position extérieure à une position intérieure, d'une désimplication à une implication.

51 Dans l'exemple (8), il est à nouveau question des différences dans l'apparence chez les femmes allemandes et les femmes françaises :

\begin{tabular}{|l|l|l|l|}
\hline (8) & LF1 & c'est vrai que enfin en moyenne il y a une grosse moyenne quand même qui \\
\hline & LF2 & qui fait attention \\
\hline & LF1 & $\begin{array}{l}\text { qui fait attention je pense qu'on fait beaucoup plus attention à notre apparence c'est } \\
\text { beaucoup plus cultivé }\end{array}$ \\
\hline
\end{tabular}

Alors que LF1 et LF2 se situent dans leurs premiers énoncés comme « informatrices » à l'extérieur du groupe désigné, les femmes françaises, LF1 change de position, s'implique en employant « on » et «notre » et parle au nom du groupe « femmes » dont elle fait partie.

53 Dans d'autres cas, en revanche, le locuteur se désimplique. Il semble que ce soit le cas lorsque l'attribution risque de menacer la face négative du locuteur : 
(9) LA et je connais une Française hein et la le première truc le premier truc elle m'a/qu'elle m'a dit c'était hein elle dit quand j'ai écouté que tu es Allemand le le la première question que j'ai voulu demander c'était ton ton relation ton ton tes pensées sur la guerre c'était ça und ich weiß nicht ob das bei euch auch so ist c'est la même chose pour vous que vous voulez savoir ce que les Allemands pensent (OFAJ 89)

Le passage de l'implication à la désimplication permet au LA de se positionner en tant qu'informateur. Dans le cas d'une réponse positive de la part de ses interlocuteurs français, il n'aurait pas à répondre en son nom, comme il a été obligé de le faire dans l'expérience citée, mais au nom de la catégorie "Allemands ».

Un autre moyen de gestion consiste à modaliser les attributions afin d'en atténuer et relativiser le stéréotypage.

Ces procédés de modalisation sont également révélateurs des contradictions qui existent entre des expériences individuelles et les scripts stéréotypes, les généralisations décontextualisées (van Dijk, 1984, Moscovici, 1986).

Une manière de relativiser le stéréotypage est de rapporter le discours d'autres personnes, des locuteurs absents, en particulier lorsque l'attribution est péjorative et menace la face positive de l'interlocuteur :

(10) LA par exemple mon cousin il a jamais appris le français et quand il est allé en France pour la première fois il avait image des Français baguette et comme ça et ce sont des voleurs il est allé en voiture et il avait peur qu'on lui vole toutes ses choses parce qu'il avait déjà entendu (OFAJ 89)

Enfin, le stéréotypage peut être affaibli par le recours à la métacommunication. En effet, en (11), les énoncés métacommunicatifs sont à la fois le résultat et la preuve des activités anticipatrices du locuteur sur les réactions possibles de l'interlocuteur :

\begin{tabular}{|l|l|l|}
\hline$(11)$ & LF1 & $\begin{array}{l}\text { je sais pas pourquoi quand on me dit Allemand c'est vrai que c'est idiot comme image } \\
\text { mais bon je vois un grand blond }\end{array}$ \\
\hline & LF2 & ben moi c'est pareil \\
\hline & LF1 & avec des yeux bleus avec des sandales \\
\hline & & (OFAJ 1991) \\
\hline
\end{tabular}

Considérant que le stéréotypage peut constituer un acte menaçant, LF1 l'atténue, en amortit en quelque sorte la portée en prévenant ses interlocuteurs par l'évaluation de son propre discours. 


\section{Conclusion} stéréotypes dans des situations de contact interculturel. Moyen économique de catégorisation, le stéréotype sert, en particulier dans les premiers instants d'une telle rencontre, à faciliter le classement, l'interprétation des données de l'altérité. Puisant dans un savoir commun, mais culturellement spécifique, sur les traits, les comportements, les habitudes prototypiques de soi-même et de l'autre, les interactants co-construisent à travers un jeu complexe d'auto-attribution et d'hétéro-attribution, leur propre image identitaire ainsi que celle de l'autre.

De nombreux moyens linguistiques et discursifs tendent à créer l'information, à susciter des comportements qui confirment et perpétuent les données stéréotypées initiales. Le groupe français, tout comme le groupe allemand, était déjà investi au préalable de caractéristiques et d'attentes que les comportements réciproques ne font que confirmer et qui les justifient à leur tour. La médiatisation de la réalité par les savoirs antérieurs filtre la perception et conduit souvent à des interprétations erronées des comportements réciproques. Elle demande également aux interactants un travail important de gestion et de « ménagement » des faces réciproques.

\section{BIBLIOGRAPHIE}

BARDIN, L. (1980). L'analyse de contenu, Paris, PUF.

BERGER, P., LUCKMANN, Th. (1992). La construction sociale de la réalité, Paris, Méridiens Klincksieck.

BROWN, P., Levinson, S. (1987). Politeness. Some universals in language use, Cambridge, Cambridge University Press.

DIJK, T van (1984). Prejudice in discourse : an analysis of ethnic prejudice in cognition and conversation, Amsterdam, Benjamin.

FINK, G.L. (1993) « Réflexions sur l'imagologie, Stéréotypes et réalités nationales dans une perspective franco-allemande », in Recherches Germaniques, 23 :3-31.

FRANCESCHINI, R., OESCH-SERRA, C., PY, B. (1989/90). « Contacts de langue en Suisse. Ruptures et reconstitutions discursives du sens en situation de migration », in Langage et Société 50/51,

117-131.

GOFFMAN, E., (1973). La mise en scène de la vie quotidienne (2 vol. ), Paris, Minuit.

KERBRAT-ORECCHIONI, C. (1984). « Les négociations conversationnelles », VERBUM VII, Fascicule 2-3.

KERBRAT-ORECCHIONI, C. (1992) Les interactions verbales, t. II, Paris, Armand Colin.

LABOV W., FANSHEL, D. (1977). Therapeutic Discourse, New York, Academic Press.

Acquisition et interaction en langue étrangère, 7 | 1996 
LE PAGE, R., TABOURET-KELLER, A. (1985). Acts of identity, Cambridge, Cambridge University Press LIPPMANN, W. (1964). Die öffentliche Meinung, München.

LÜDI, G. (1995). « L'identité linguistique des migrants en question : perdre, maintenir, changer », in G. LÜDI \& B. Py, Changement de langage et langage du changement, Lausanne, Editions l'Age d'Homme.

MOSCOVICI, S. (1986). «L'ère des représentations sociales », in W. DOISE et A. PALMONARI (eds), L'étude des représentations sociales, Neuchâtel/Paris, Delachaux \& Niestlé.

OESCH-SERRA, C. (1995). «L'évolution des représentations », in G. LÜDI et B. PY, Changement de langage et langage du changement, Lausanne, Editions L'Age de l'Homme.

PERREFORT, M. (1990). Identité, compréhension et malentendus dans la communication interculturelle à l'exemple franco-allemand, Thèse, Besançon, 1990.

PERREFORT, M. (sous presse). Lettres de poilus franc-comtois, Besançon, Ed. Cêtre.

QUASTHOFF, U. (1973). Soziales Vorurteil und Kommunikation : Eine sprachwissenschaftliche Analyse des Stereotyps, Frankfurt/Main, Fischer Athenäum.

SACKS, H. (1972). « On the Analyzability of Stories by Children », in J. GUMPERZ, D. HYMES, (eds.) Directions in Sociolinguistics. The Ethnography of Communication, New York, Holt, 325-342.

TAJFEL, H. (1982). Gruppenkonflikt und Vorurteil : Entstehung und Funktion sozialer Stereotypen, Berlin/Wien, Hans Huber.

\section{NOTES}

1. On reproduit bien sûr fidèlement ici l'orthographe de ces lettres.

\section{RÉSUMÉS}

Dans le contact interculturel le savoir quotidien perd son efficacité explicative et la nécessité s'impose de reconstruire des significations. Dans ces opérations d'interprétation, le stéréotype remplit une fonction sémiotique importante. En référence à Berger et Luckmann (1992) le stéréotype est traité en tant qu'objet de discours, actualisé et géré par les interactants. Pour saisir la construction discursive du stéréotype, on analyse des conversations entre Français et Allemands lors de rencontres binationales. L'analyse décrit quelques formes de stéréotypage et montre que le recours au stéréotype fonctionne également comme marqueur d'identité et, corrélativement, d'altérité.

We follow Berger and Luckmann (1992) in considering the stereotype as a discourse object which is actualised and managed by the speech partners. We examine conversations between French and German participants in bi-national meetings in an attempt to trace the discourse construction of the stereotype, analysing different types of stereotype, and demonstrating that use of stereotypes also functions as an identity marker and, correspondingly, as a marker of 
otherness. We propose that it is because everyday encyclopedic knowledge is less functional in intercultural interaction that the steriotype comes to assume an important semiotic function.

\section{AUTEUR}

\section{MARION PERREFORT}

Université de Franche-Comté 